\title{
ENDOSCOPIC TRANSCANAL MYRINGOPLASTY-SMART MYRINGOPLASTY FOR NEX GEN ENT SURGEONS: OUR EXPERIENCE
}

\author{
Alok Kumar ${ }^{1}$, Meshram Namdeorao $^{1}$, and Ajay Gupta ${ }^{1}$ \\ ${ }^{1}$ Tata Main Hospital
}

May 19, 2020

\begin{abstract}
INTRODUCTION Otolaryngology is one of the branch where endoscopes is being widely used nowadays to perform different surgeries. Ear surgeries are of special interest among the ENT surgeons. It has several advantages compared to the microscopes. METHODS 60 patients underwent endoscopic transcanal myringoplasty by the postgraduate residents under direct supervision of same consultants, using temporalis facia graft. RESULTS The overall success rate in terms of graft uptake was $86.67 \%$ and the AB gap closure of $<10$ DB in $63.3 \%$ of cases and 10-20 DB in 33.3\%. CONCLUSION Endoscopic myringoplasty was found to be equally effective, less morbid and very cost effective than the microscopic myringoplasty. This was applicable irrespective of the size of the perforation and condition of the middle ear(Dry/Wet) in our centre
\end{abstract}

\section{INTRODUCTION}

Since the 1950's microscopes have become the standard technique for central perforation closure and had revolutionized the outcome of the surgery. With time, it was realized that with microscopes it was difficult to operate in case of anterior bony overhang. It was not possible to look into every nook and corners of the middle ear; the anterior tucking was difficult in a very thin anterior remnant. The only advantage the microscope has is that one can use two hands for better control during graft manoeuvre. Endoscopes have fared better in terms of visualisation but for better control with a single hand one needs practice and follow some important technical improvisation specially for the residents.

\section{AIMS AND OBJECTIVES}

The aim was to evaluate the result of endoscopic myringoplasty in terms of the graft uptake rate, visualisation of the middle ear, and reduction in the $\mathrm{AB}$ gap and to validate it with the results of microscopic myringoplasty

\section{MATERIAL AND METHOD}

This was a prospective study carried out between January 2019 to January 2020.

\section{INCLUSION CRITERIA}

1. Patient with $\mathrm{H} / \mathrm{O}$ ear discharge

2. All Tubo- tympanic diseases

3. Young adults to age up to 70 years

4. Dry/ mucoid / mucopurulent discharge

5. Conductive, Mixed or Sensorineural hearing loss

EXCLUSION CRITERIA 
1. Attico antral disease

2. Ossicular chain dislocation

3. Ossicular chain fixation

\section{METHODS}

All patients with history of ear discharge were given a course of Tab Amoxycillin + Clavulanic acid $625 \mathrm{mg}$ three times daily for five days. Aural swab was sent for culture and sensitivity. Pure Tone audiometry and Eustachian tube function test was done in all cases. All cases underwent X-ray Mastoid (Lateral Oblique view). The antibiotic was switched as per the culture and sensitivity report and continued for next five days, although dryness of ear was not a criterion for surgery. Patients underwent Endoscopic myringoplasty with intravenous antibiotic, oral antihistamine one day prior to surgery in wet ear cases. The patients were followed up one week, one month and three months post-surgery. Endoscopic examination of the patient post one month and three months. Intact graft after 1 month of surgery was taken as anatomical success ${ }^{[1]}$. Audiometric evaluation was done on third month post op.

\section{PROCEDURE}

All cases were done under general anaesthesia. Rigid endoscope $0^{0}, 2.5 \mathrm{~mm}$ was used to visualize the tympanic membrane. We had used the same microsurgery instrument that were used for Microscopic Myringoplasty. Patient were laid in supine position, head rotated to the opposite side and supported with a head ring. Separate incision, post auricular just adjacent to the hairline measuring 2.5 to $3 \mathrm{~cm}$ was given to harvest the Temporalis facia. The graft was "paper dried". All cases were supervised by a single surgeon and performed by postgraduate residents.

\section{TECHNIQUE}

The external auditory canal was infiltrated with $2 \%$ 1:200,000 xylocaine with adrenalin using Insulin syringe just distal to the hairy part along the circumference until one can notice the blanching of the skin. A maximum of $5 \mathrm{ml}$ was used for infiltration. We had used ample of adrenalin soaked cotton balls to achieve haemostasis and in all cases, we have achieved bloodless field which is very important for endoscopic myringoplasty other than the technicality. Tarabichi suturing at the concha was done to straighten the cartilaginous external auditory canal. All cases were done with surgeon in sitting position with the elbow of the endoscope holding hand resting on the table which provided stability. Margins of the Tympanic membrane remnant was freshened with curved pick or sickle knife. Tympanomeatal flap raising was tailor made as per the site of the Tympanic membrane perforation as illustrated in the diagram below. Diagram1 depicting a perforation in the anterior quadrant with sufficient anterior margin, here an anterior tunnel was created just close to the annulus with Tympanomeatal flap raised from 12 'o'clock to 6 'o'clock and the part of the graft was taken out from the tunnel to prevent anterior blunting. Diagram 2 a condition where the perforation was in the posterior quadrant, here the tympanomeatal flap was raised from the 12'o'clock to 6'o' clock and the graft was placed medial to the malleus. Diagram 3 showing a subtotal perforation with very thin anterior remnant, here the Tympanomeatal flap was raised from 11'o' clock round the circumference to 2 'o' clock and a big graft was placed resting over the bony canal throughout the circumference. The eustachian tube opening was packed with gelfoam and gelfoam bed was prepared over the middle ear. The dried graft was placed over the gelfoam bed and was laid throughout the circumference of the bone external canal. The tympanomeatal flap was reposed back and again supported with a layer of gelfoam.

\section{STATISTICAL ANALYSIS}

All the data would be selected randomly and tabulated, and then analysed with appropriate statistical tools "Med Calc". Data will be presented as mean with standard deviation or proportions as appropriate. Mean, median, standard deviation and variance would be calculated and following statistical significance tests would be applied. Abnormally distributed data were expressed as mean and standard deviation. Categorical variables were expressed as frequencies and percentage or relative values.

1. Statistical analysis would be done using "Chi - square Test" for categorical variables. 
2. Odds Ratio (OR) with $95 \%$ class interval also calculated.

A "p-value" should be considered to be non-significant if $>0.05$ and significant if $<0.05$.

Statistical methods would be used to find the significance of homogeneity of study characteristics between the two groups of patients. Their inference will be as follows-

$\mathrm{P}>0.05$ statistically insignificant

$\mathrm{P}<0.05$ statistically significant

$\mathrm{P}<0.01$ statistically highly significant

$\mathrm{P}<0.001$ statistically very highly significant

\section{RESULTS}

Out of 60 patients the majority belonged to the age group 41-50 years. Females were more commonly affected than males. Right side was more commonly involved than the left side. Myringoplasty was performed in both the dry as well as wet middle ear and the uptake was $86.67 \%$,Figure 1 . Two quadrant perforation had the maximum graft uptake and overall the $\mathrm{P}$ value was $<0.0001$,Table 1 .Air-Bone gap post op status was $>10 \mathrm{DB}$ in 38 patients $(\mathrm{P}$ value -0.0035$), 10-20 \mathrm{DB}$ in 20 patients $(\mathrm{P}$ value-0.1373) and $>20$ DB in 2 patients(P value-0.0331) Table 2 .Causes of Failure was analysed and it was found that none of the patients has Anterior Blunting. In three patient's pre-op discharges might be the cause of failure. Three patients gave a history of exposure to loud sound and in the remaining three the ET dysfunction might be the cause.

The Table 3. shows the association between Graft success rate and the various prognostic factors. The pre-op and post op photograph has been shown, Picture 1 .

\section{DISCUSSION}

The major difference between Microscopy and Endoscopy is the surgical view. Tarabichi et al reported that the view during microscopic surgery is defined and limited by the narrow segment of the ear canal ${ }^{[2,3]}$. By contrast transcanal endoscope bypasses the narrow segment of the ear canal and provides a wide view with 00 endoscopes. Furukawa et al ${ }^{[4]}$, Lade et al ${ }^{[5]}$ and Harugop et al ${ }^{[6]}$ conducted studies to compare the microscopic and endoscopic views tympanoplasty. They reported that in the microscopy groups, the tympanic annulus was not completely visualized in $17 \%$ to $20 \%$ of patients, thus requiring canalplasty. However, in the endoscopy groups, the tympanic annulus was completely visualized; hence, no patient required canalplasty. Ayache ${ }^{[7]}$ reported an even higher rate of $73 \%$ of patients in whom anterior perforations of the tympanic membrane were poorly visualized.

Comparing the success rates of microscopic and endoscopic tympanoplasty is often the main concern. Success rates of $90 \%$ to $95 \%$ for microscopic myringoplasty have been reported ${ }^{[8,9]}$. Similar success rates of $80 \%$ to $100 \%$ have also been reported for endoscopic myringoplasty ${ }^{[10,11,12]}$ Harugop et al and Lade et al compared the success rates of microscopic and endoscopic myringoplasty and reported no difference. The success rate in our study was $86.6 \%$ and the $\mathrm{AB}$ reduction to $<10$ was in $63.3 \%$ and $10-20$ DB reduction was $33.3 \%$ of patients. In 2008, Satyawati Mohindra et al. did 49 cases of myringoplasty and 6 cases of Ossiculoplasties through the transcanal route using rigid endoscopes. The success rate regarding perforation closure was $91.5 \%$ and average air bone gap improvement was $22.24 \mathrm{~dB}$ in the myringoplasty groups [13]. Ahmed ELGuindy (Tanta, Egypt) has evaluated the role of the rigid endoscope in the management of 36 cases of dry central perforation of the tympanic membrane. The graft uptake rate was $91.7 \%$ and air bone gap was closed to less than $10 \mathrm{~dB}$ in $83.3 \%$ [14].

Endoscopic myringoplasty carries several advantages over the Microscopic myringoplasty, most important it carries minimal scar which is especially beneficial for the females. Shaving is not required in any of these cases. Endoscopy has several disadvantages in ear surgery compared with microscopy. First, the endoscope must be held in one hand, and only the other hand is free to operate; this procedure is particularly cumbersome when bleeding obscures the view of the operating field. In addition, endoscopy provides a 
monocular view, which causes the loss of depth perception compared with the binocular view provided through microscopy. Moreover, endoscopic myringoplasty still requires more training experience ${ }^{[2,3,11]}$. We partially disagree with the above statement as most of the endoscopic surgeries are done single handed so the required expertise is nothing special for endoscopic myringoplasty. To us there are two most important requirement others than the better hand control, one is a bloodless which is achieved by sufficient canal infiltration with 1:2 Lakh Xylocaine with Adrenalin and the other is dry temporalis fascia graft which makes the manoeuvre of the graft easier. As far as the learning curve of the post graduates is concerned its same as the other endoscopic procedure requirement i.e. practice of diagnostic endoscopy in every patient in the OPD. Temporal bone dissections to learn the methods of myringoplasty before doing actual myringoplasty. In our study, endoscopes allowed us close inspection and photo documentation of the tympanic membrane perforation, the drum remnant, the eustachian tube orifice, the middle ear mucosa and ossicular chain. The anatomical variations (tortuous or stenotic ear canal, anterior meatal overhang etc. that hamper the view of entire tympanic membrane during ear surgery were overcome by the use of endoscopes. Moreover, the permeatal route avoided post aural incision, resulting in less operative time and postoperative pain and morbidity. Disadvantages of the endoscopic myringoplasty included the one-handed surgical technique, a loss of depth perception, limited magnification and the need for training ${ }^{[15]}$. One major safety concerns with endoscopic ear surgery is excessive heat dissipation. This was evident only when a xenon light source was used. Adequate illumination of the middle ear space can be accomplished with lower settings on the regular light source (because of the size of the cavity) without the need of the xenon systems. Also, the tip of the endoscope requires continuous cleaning with anti-fog solution, which probably helps in cooling the endoscope

The endoscopic technique is the future of myringoplasty and tympanoplasty. With results similar to the microscopic techniques and the added advantages, the endoscope may become more popular than the microscope. The endoscope holds the greatest promise in tympanoplasty and cholesteatoma surgery and should increase the utilization of transcanal over post-auricular procedures ${ }^{[16,17]}$.

\section{CONCLUSION}

Panoramic, wide angle, and magnified view provided by endoscope as well as ability to easily negotiate through EAC and uninterrupted picture overcomes most of the disadvantage of microscope. In our study success rate was comparable between endoscopic and microscopic technique. In terms of morbidity and postoperative recovery, endoscope produced better results. Loss of depth perception and one-handed technique are some of the disadvantage of endoscope that can be overcome with practice and use of endo-holder. Thus, endoscopic myringoplasty can be a good alternative of microscopic myringoplasty.

\section{CONFLICT OF INTEREST}

The authors declare that they have no conflict of interest

\section{INFORMED CONSENT}

Informed consent was obtained from all individual participants included in the study.

\section{REFERENCES}

1. Arindam Das et al.Myringoplasty: Impact of Size and Site of Perforation on success rate.Indian J Otolaryngol Head and Neck Surgery.2015 June; 67(2): 185-189.

2. Tarabichi M. Endoscopic middle ear surgery. AnnOtolRhinolLaryngol.1999;108(1):39-46.

3. Tarabichi M, Ayache S, Nogueira JF, Al Qahtani M, Pothier DD. Endoscopic management of chronic otitis media and tympanoplasty. Oto laryngol Clin NorthAm.2013;46(2):155-163.

4. Furukawa T, Watanabe T, Ito T, Kubota T, Kakehata S. Feasibility and advantages of transcanal endoscopic myringoplasty. OtolNeurotol.2014;35 (4):e140-e145.

5. Lade H, Choudhary SR, Vashishth A. Endoscopic vs microscopic myringoplasty: a different perspective. Eur Arch Otorhinolaryngol. 2014;271(7):1897-1902.

6. Harugop AS, Mudhol RS, Godhi RA. A comparative study of endoscope assisted myringoplasty and micrsoscope assisted myringoplasty. Indian J Otolaryngol Head Neck Surg. 2008;60(4):298-302. 
7. Ayache S. Cartilaginous myringoplasty: the endoscopic transcanal procedure. Eur Arch Otorhinolaryngol.2013;270(3):853-860.

8. Sheehy JL, Anderson RG. Myringoplasty: areviewof472cases.AnnOtolRhinolLaryngol. 1980;89(4, pt1):331-334.

9. Rizer FM. Overlay versus underlay tympanoplasty, II:thestudy.Laryngoscope.1997; 107(12,pt2):26-36. 4. Applebaum EL, Deutsch EC. An endoscopic

10. el-Guindy A. Endoscopic transcanal myringoplasty. JLaryngolOtol.1992;106(6):493-495

11. . Karhuketo TS, Ilomäki JH, Puhakka HJ. Tympanoscope-assisted myringoplasty. ORLJ OtorhinolaryngolRelatSpec.2001;63(6):353-357

12. Yadav SP, Aggarwal N, Julaha M, Goel A. Endoscope-assisted myringoplasty. Singapore Med J.2009;50(5):510-512.

13. Mohindra S, Panda NK. Ear surgery without microscope; is it possible. Indian J Otolaryngol Head Neck Surg. 2010;62(2):138-141. doi: 10.1007/s12070-010-0033

14. EL-Guindy A. Endoscopic transcanal myringoplasty. J Laryngol Otol. 1993;106:493-495. doi: $10.1017 /$ S0022215100119966.

15. Glasscock ME, Shambaugh GE (2003) Tympanoplasty. In: Glasscock and Shambaugh, surgery of the ear, 5th edn. People's Medical Publishing House, Connecticut, pp 350-370

16. Raj A, Meher R. Endoscopic transcanal myringoplasty - a study. Indian J Otolaryngol head neck surg. 2001;53(1):47-49. doi: 10.1007/BF02910979.

17. Mc Kennan KX. Endoscopic second look mastoidoscopy to rule out residual epitympanic - mastoid cholesteatoma. Laryngoscope. 1993; 103:810-814

\section{Hosted file}

GRAPHS.docx available at https://authorea.com/users/323880/articles/452325-endoscopictranscanal-myringoplasty-smart-myringoplasty-for-nex-gen-ent-surgeons-our-experience

\section{Hosted file}

TABLES.docx available at https://authorea.com/users/323880/articles/452325-endoscopictranscanal-myringoplasty-smart-myringoplasty-for-nex-gen-ent-surgeons-our-experience 


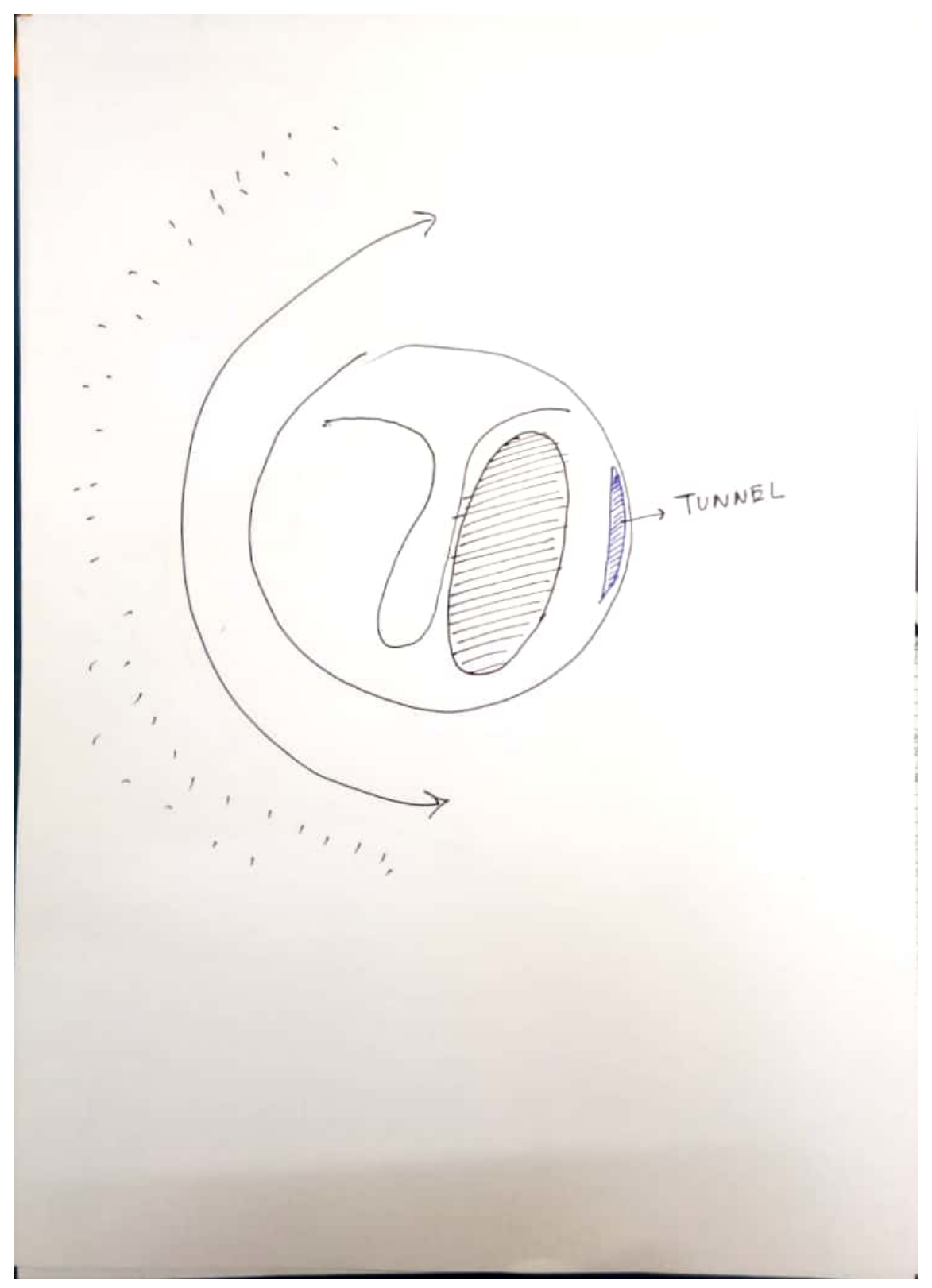




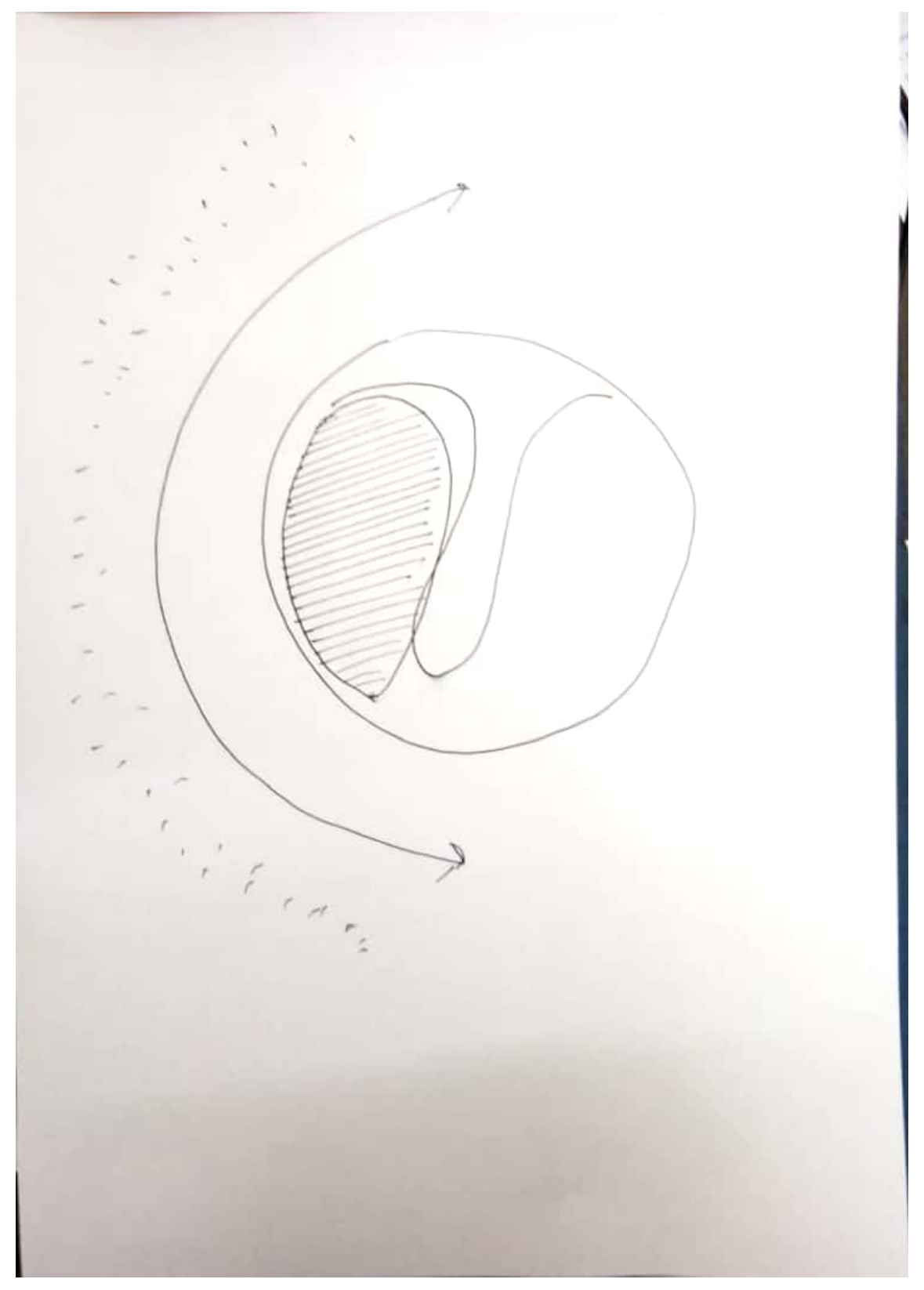




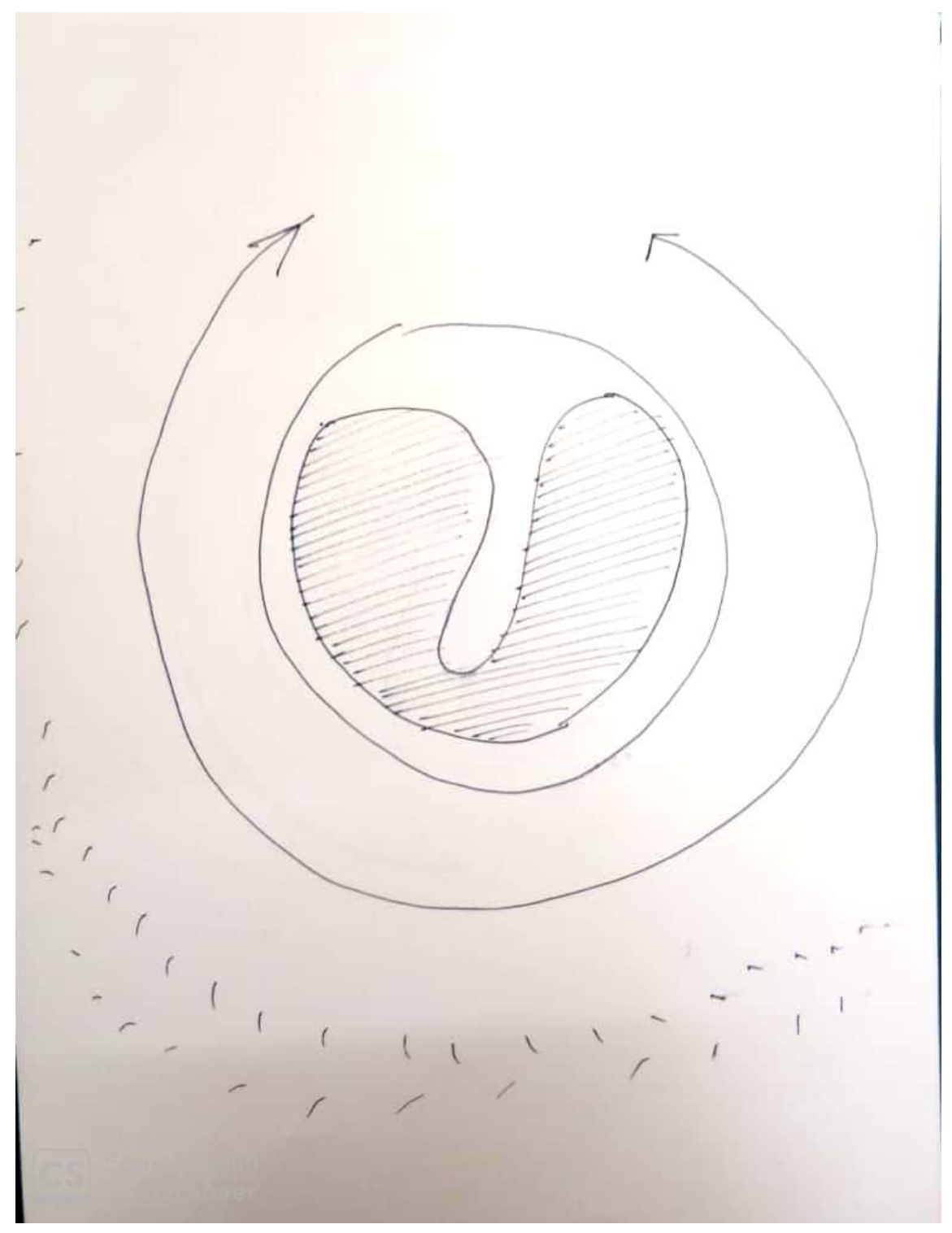




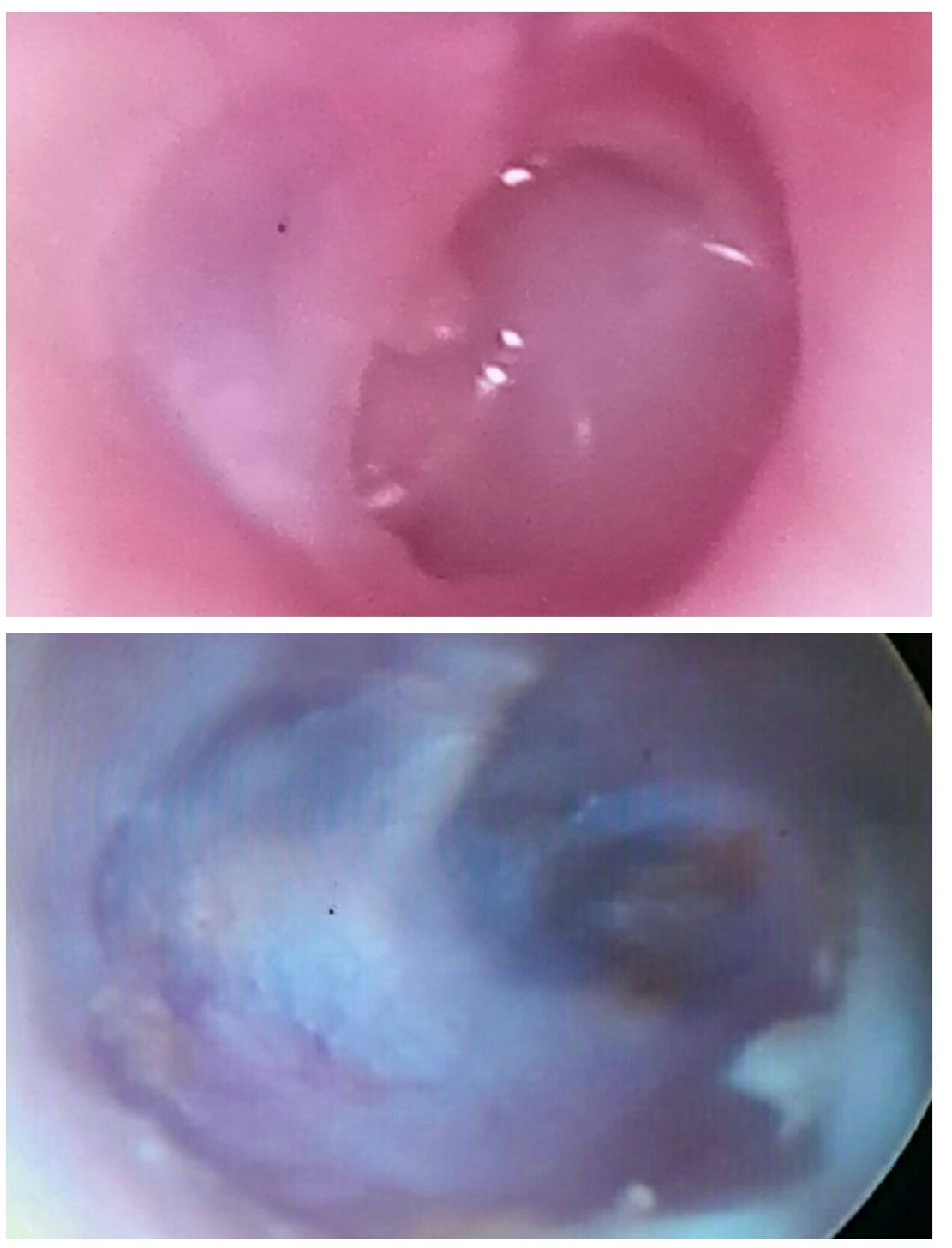

